\title{
Referee acknowledgement
}

The Editors should like to thank all scientists who supported the journal by serving as additional referees for articles submitted in 1985:
A. Aeschlimann, Neuchâtel
P. Andrews, Wuppertal
W. Becker, Hamburg
W. Böckeler, Kiel
G. Brugerolle, Aubiere
H.J. Bürger, Hannover
D.W. Büttner, Hamburg
K.P. Chang, Chicago
R.M. Cable, Lafayette
A.W. Cheever, Bethesda
B. Chobotar, Berrien Springs
C. Crandall, Gainesville

P. D'Alesandro, New York

J. Diamantstein, Berlin

P.A. Diehl, Neuchâtel

J. Dönges, Würzburg

D. Düwel, Frankfurt-Höchst

D.M. Dwyer, Bethesda

F. Ebert, Hamburg

B. Enders, Marburg

R. Entzeroth, Bonn

H. Feldmeier, Berlin

C.D. Fitch, St. Louis

W. Frank, Stuttgart-Hohenheim

M. Franz, Hamburg

M.K. Grieshaber, Münster

E. Greuel, Bonn

J. Grunewald, Tübingen

W. Haas, Frankfurt

A. Haberkorn, Wuppertal

M. Hauser, Bochum

H.-G. Heidrich, Martinsried

A.O. Heydorn, Berlin

R. Herman, Piscataway

$F$. Hörchner, Berlin

K. Janitschke, Berlin

F. Kierszenbaum, East Lansing

E. Königk, Hamburg

W. Körting, Hannover

H.E. Krampitz, München

G. Kümmel, Karlsruhe

W. Kunz, Düsseldorf
J. Lamina, München

M.J. Lavoipierre, Davis

B. Loos-Frank, Stuttgart

W. Maier, Bonn

D. McLaren, London

K. Meier-Brook, Tübingen

H. Möller, Kiel

G. Mosbacher, Saarbrücken

H. Mühlpfordt, Hamburg

W. Nachtigall, Saarbrücken

F.R. Opperdoes, Brüssel

R. Overstreet, Ocean Springs

W. Peters, Düsseldorf

W. Pflüger, Leverkusen

G. Piekarski, Bonn

W. Raether, Frankfurt

H. Reichenbach-Klinke, München

H.J. Risse, Berlin

L.S. Roberts, Lubbock

F. Römer, Nidau

M. Rommel, Hannover

A. Ruthmann, Bochum

G. Schaub, Freiburg

E. Schein, Berlin

K. Schnepf, Heidelberg

A. Scholl, Bern

J. Schottelius, Hamburg

C. Spatz, Freiburg

C.A. Speer, Montana

K.D. Spindler, Düsseldorf

H. Tanowitz, Bronx

H. Taraschewski, Bochum

G.A.T. Targett, London

D.W. Taylor, Washington

J.E. Übelacker, Dallas

J. Völker, Hamburg

R.D. Walter, Hamburg

E.C. Weinbach, Bethesda

$P$. Wenk, Tübingen

H. Wetzel, München

F. Wunderlich, Freiburg

H. Zahner, Gießen 